\title{
EULAR COVID-19 Recommendations
}

Rachel Donnison

Editorial Assistant

Citation: EMJ Rheumatol. 2020;7[1]:19-21.
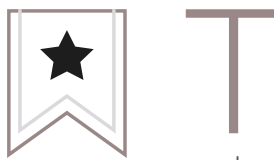

REATING patients with autoimmune diseases, the rheumatology community is naturally concerned with the spread of COVID-19; as Prof Robert Landewe of the University of Amsterdam, Amsterdam, the Netherlands stated: "immunosuppression and infection do not go along very well." On April $3^{\text {rd }}$ 2020, EULAR President-Elect Prof Hans Bijlsma founded a task force to create a comprehensive set of guidelines for clinicians treating patients with rheumatic disease and COVID-19, though not in a typical manner. Using Microsoft Teams and teleconferences, the newly founded committee set out to create a comprehensive set of recommendations. Time was of the essence, as the virus continued to spread and rheumatologists looked to EULAR for guidance. Exactly 3 months later the guidelines were presented at the EULAR 2020 virtual congress on $3^{\text {rd }}$ June 2020.

\section{PROVISIONAL RECOMMENDATIONS}

Musculoskeletal disease guidelines in the context of severe acute respiratory syndrome coronavirus-2 (SARS-CoV-2), as outlined by EULAR, is "an unprecedented set of recommendations," according to Prof Landewé. In a pandemic, EULAR's usual methodical approach to finalising recommendations, which takes at least 12-18 months, had to be significantly shortened; the stages of consensual approach and systematic literature research were forgone as there was no literature or evidence to guide them.

The recommendations for patients with rheumatic disease and COVID-19 start with five overarching principles:

1. There is no evidence that these patients are more at risk of contracting SARS-CoV-2, nor do they have a worse prognosis if they are infected.

2. Diagnosis and treatment of patients is the primary responsibility of an expert in treating COVID-19 (e.g., a respiratory physician or infectious disease specialist).

3. Decisions based on immunosuppressive treatment (e.g., disease-modifying antirheumatic drugs [DMARD]), maintenance, or discontinuation should involve rheumatologists.

4. Rheumatologists should be involved in local, regional, or national guideline committees regarding use of DMARD, the use of which should be a multidisciplinary decision.

5. Off-label use of DMARD in COVID-19 outside the context of clinical trials should not be encouraged.

Prof Landewé concluded by highlighting "the current evidence is extremely sparse and fragmented" and that as a task force they are "flying blindly," whilst also following many jurisdictions within Europe, with many conflicting opinions. 


\section{ACR RECOMMENDATIONS}

The American College of Rheumatology (ACR) recommendations were subsequently presented by the Chair of the ACR COVID-19 Clinical Task Force Prof Ted Mikuls of the University of Nebraska Medical Center, Omaha, Nebraska, USA. To accommodate the changing literature and evidence landscape regarding the virus, the ACR task force has committed to a monthly update of the recommendations, compared to EULAR's quarterly pledge. Voting initially on 81 statements, of which 77 were approved, the team combined these into a list of 25 guidance statements, compared to EULAR's 13.

The ACR recommendations are divided into three groups, the first being guiding principles with a primary focus on the patient and provider level, based on the sparse but rapidly evolving evidence. The second grouping of ACR guidance concentrates on stabilising patients: "In the absence of known exposure and the absence of COVID-19 infection, our panel felt very strongly about the importance of continuing rheumatic disease treatments," conveyed Prof Mikuls. The overarching theme of this second group was the potential risk that unchecked inflammation and rheumatic disease posed to patients with COVID-19.

Finally, the third grouping provided guidance to physicians for patients with known exposure or presumed infection of SARS-CoV-2. Prof Mikuls was careful to point out that "our recommendations suggest at least temporary discontinuation of most immunosuppressive and biologic medications" while patients recover from infection.

Though tasked with describing the differences between the EULAR and ACR recommendations, Prof Mikuls found the similarities reassuring: "we're approaching the unknown from very

"the current

evidence is extremely sparse and fragmented" different parts of the world, and arriving in a very similar place." 
